\title{
Vesta Sarkhosh Curtis, Elizabeth J. Pendleton, Michael Alram, Touraj Daryaee (eds.). The Parthian and Early Sasanian Empires: Adaptation and Expansion. Proceedings of a Conference held in Vienna, 14-16 june 2012
}

\section{Rémy Boucharlat}

\section{OpenEdition}

Édition électronique

URL : http://journals.openedition.org/abstractairanica/47013

DOI : 10.4000/abstractairanica.47013

ISBN : 1961-960X

ISSN : 1961-960X

Éditeur :

CNRS (UMR 7528 Mondes iraniens et indiens), Éditions de l'IFRI

Référence électronique

Rémy Boucharlat, « Vesta Sarkhosh Curtis, Elizabeth J. Pendleton, Michael Alram, Touraj Daryaee (eds.). The Parthian and Early Sasanian Empires: Adaptation and Expansion. Proceedings of a Conference held in Vienna, 14-16 june 2012 », Abstracta Iranica [En ligne], Volume 37-38-39 | 2018, document 67, mis en ligne le 30 décembre 2018, consulté le 02 octobre 2020. URL : http://journals.openedition.org/ abstractairanica/47013 ; DOI : https://doi.org/10.4000/abstractairanica.47013

Ce document a été généré automatiquement le 2 octobre 2020.

Tous droits réservés 


\title{
Vesta Sarkhosh Curtis, Elizabeth J. Pendleton, Michael Alram, Touraj Daryaee (eds.). The Parthian and Early Sasanian Empires: Adaptation and Expansion. Proceedings of a Conference held in Vienna, 14-16 june 2012
}

\author{
Rémy Boucharlat
}

\section{RÉFÉRENCE}

Vesta Sarkhosh Curtis, Elizabeth J. Pendleton, Michael Alram, Touraj Daryaee (eds.). The Parthian and Early Sasanian Empires: Adaptation and Expansion. Proceedings of a Conference held in Vienna, 14-16 june 2012. Oxford \& Philadelphia, Oxbow, 2016, X-132 p., ill (BIPS, Archaeological Monograph Series)

1 Les quatorze contributions publiées ici sont le fruit d'une conférence tenue à Vienne en relation avec la Sylloge Nummorum Parthicorum, mais pour le colloque la chronologie a été étendue au début de l'époque sassanide. Les éditeurs notent que l'époque parthe reste sous-estimée en Iran. Ce constat ne résulte pas d'un préjugé ou d'un a priori des spécialistes, mais, selon moi, simplement de la rareté des sites identifiés de cette période. Hasard ou défaut d'identification? La question reste entière en ce début du XXIe siècle. Les contributions correspondent parfaitement au cadre chronologique du titre de l'ouvrage.

2 Les articles sont regroupés selon quatre sections. Historical [and religion]: Jérome Gaslain, « Some Aspects of Political History, Early Arsacids Kings and the Seleucids », en peu de pages, l'A s'inscrit dans la tendance de l'historiographie moderne en 
reconnaissant aux Parthes et à leurs souverains du IIIe s. av.n.è. une véritable politique et une organisation dans un territoire défini, bien éloignées de celle de tribus nomades. M. Rahim Shayegan, "The Arsacids and Commagene" analyse finement les déclarations de Mithridate VI (selon Trogue Pompée), roi du Pont au $1^{\mathrm{er}}$ s. av.n.è. et celle d'Antiochos I de Commagène sur le haut-lieu du Nemrud Dağ plus tard dans ce siècle, se prétendant l'un et l'autre descendants des Achéménides par leur père et d'Alexandre par leur mère. De leur côté, les rois arsacides ont très tôt repris la titulature achéménide de « roi des rois » à la fin du $2^{\mathrm{e}} \mathrm{s}$. av.n.è. Au-delà du prestige du titre ou de l'ascendance, ces déclarations servent aussi ou d'abord à justifier les revendications territoriales. Marek Jan Olbrycht, « Dynastic Connections in the Arsacid Empire and the Origin of the House of Sāsān » remonte dans les origines d'Ardeshir I dans les familles des satrapies orientales et parmi les descendants de Gondophares. Si les sources nous apparaissent complexes ou contradictoires, c'est parce que les premiers Sassanides ont souvent manipulé leur ascendance entre Sasan et Papag. Touraj Daryaee, «From Terror to Tactical Usage: Elephants in the Partho-Sasanian Period » Depuis les Achéménides jusqu'aux Sassanides, les éléphants sont utilisés dans l'armée comme des "tanks ", avant d'être finalement réduits à être des observatoires surélevés pour les chefs de l'armée. Mais dès le $1^{\mathrm{er}} \mathrm{s}$. de n.è., sous les Parthes, l'éléphant est aussi une représentation de la royauté comme le montre le revers de monnaies. Mehrdad Ghodrat-Dizaji, «Remark on the Location of the Province of Parthia in the Sasanian Period » montre que la Parthie, localisée dans le nord et nord-est de l'Iran, à l'est de la mer Caspienne à l'époque achéménide, s'étend dès les Parthes au sud de l'Elborz (l'A aurait pu citer le site de Shahr-i Qumis comme exemple), puis bien au-delà au sud, englobant sans doute la région d'Ispahan et même vers le NO de l'Iran. Andrea Gariboldi, "The Birth of the Sasanian Monarchy in Western Sources" donne des sources romaines, textes et monnaies, qui citent les premiers Sassanides tantôt comme Perses, tantôt comme Parthes. Les auteurs des textes sont conscients que ceux-ci préfèrent être distingués des Parthes, mais les Romains ne connaissent pas l'origine de la dynastie. Antonio Panaino, "Kirdēr and the Re-Organisation of Persian Mazdeism » souligne les interactions entre politique et religion dans les paroles de Kirdēr qui décrit son rôle dans le contrôle des territoires qui recouvrent la communauté des clercs mazdéens.

3 Linguistic: Rika Gyselen, «The Parthian Language in Early Sasanian Times» rappelle que si la langue parthe est encore employée dans les inscriptions des premiers Sassanides (la Ka'ba-i Zardusht, Paikuli). Elle se maintient aussi au travers de plusieurs sceaux sûrement d'époque sassanide, mais son emploi cesse probablement au cours du IV ${ }^{e}$ siècle. Khodadad Rezakhani, «From Aramaic to Pahlavi : Observations Based on the Persis Coin Series» enquête sur l'origine de l'écriture moyen-perse/pahlavi sous Shapur et Ardeshir dans les émissions vers 200-210, et remonte plus avant avant en étudiant des caractères et la paléographie sur des monnaies de Perside. Finalement, une origine dans le monde indo-saka reste une possibilité. Daryoosh Akbarzadeh, «Parthian Studies: Two New Notes » traite de deux termes moyen-perses employés à l'époque parthe à partir de textes très postérieurs de l'époque médiévale.

4 Archaeological: Antonio Invernzzi, "A note on Architectural Traditions in Arsacid Parthia: The Round Hall at Nisa » revient sur la reconstitution de la couverture de la Salle ronde de Nisa, éliminant définitivement l'hypothèse d'un agencement pyramidal de poutres en bois, qu'il remplace par un dome surhaussé reposant directement sur le sol, une forme à comparer à la couverture d'ossuaires d'Asie centrale dès le Ier mill. 
av.n.è. Barbara Kaim, «Women Dance and the Hunt : Splendour and Pleasures at Court Life in Arsacid and Early Sasanian Art ", s'interroge sur la signification de certains thèmes religieux (dionysiaques par exemple, ou dieu Pan, etc.) dans les scènes de réjouissance ou de banquet comme dans les stucs de Qal'eh-e Yazdgerd, à la fin de l'époque parthe ou les mosaïques de Bishapur au IIIe s. de n.è. Sont-ils utilisés seulement comme thèmes décoratifs? (cf. cr. Ce volume Barbara Kaim, « Women Dance and the Hunt... »).

5 Numismatics: Pierre-Yves Boillet, "Quantifying Monetary Production: Ecbatana and Media in Parthian Times » L'A, spécialiste de l'atelier d'Ecbatane (voir son importante thèse citée en référence) montre que la production monétaire $d$ 'Ecbatane diminue sous les Parthes pour la production des bronzes mais augmente pour les drachmes, tandis que rien n'indique un déclin des activités commerciales des Séleucides aux Parthes. Nikolaus Schindel, "The Coinage of Paradan and Sind in the Context of Kushan and Kushano-Sasanian Numismatic " se situe à l'autre extrémité (orientale) du monde sassanide. À partir de la présence des rubans sur les bustes des monnaies du Paradan (principauté du sud-ouest du Pakistan) aux IIe et IIIe s. de n.è., l'A remet indirectement en cause le début de l'ère Kanishka, après 224 de n.è. au lieu de 127, date majoritairement acceptée. L'autre sujet est le monnayage du Sind qui est particulier à cette province, montrant que cette région n'était pas une province sassanide comme celles de l'intérieur de l'empire.

\section{AUTEURS}

\section{RÉMY BOUCHARLAT}

UMR 5133 CNRS-Université de Lyon 